\title{
Der Selbstmord im Felde.
}

\author{
Von \\ Dr. med. Max Sichel.
}

(Eingegangen am 11. Mai 1919.)

Die alte Erfahrung, daß in Kriegszeiten die Geistesstörungen an Zahl zunehmen, die Selbstmordziffer hingegen sinkt, dürfte im großen und ganzen auch durch die Ereignisse der vier vergangenen Jahre ihre Bestätigung erhalten. Zahlenmäßige Unterlagen werden erst zu erhalten sein, wenn das ungeheure Material gesichtet und sachgemäß verarbeitet werden kann. Infolge der Heranziehung von Massen zum Heeresdienst, wie sie in gleicher Zahl kein Krieg der Vergangenheit jemals erfordert hat, sowie der durch den Zwang der Verhältnisse gebotenen Erweiterung des Tauglichkeitsbegriffs mußte von vornherein mit einer starken Zunahme der psychischen Morbidität gerechnet werden. Die Ausmerzung geistig minderwertiger Elemente, wie sie in Friedenszeiten geüht wurle, war bei den Ansprüchen dieses Krieges nicht durchführbar; hieraus erklärt sich vor allen Dingen die große Zahl der sogenannten Kriegsneurosen, die letzten Endes nichts anderes sind, als Reaktionen psychisch labiler Individuen auf unlustbetonte Situationen. Die Abnahme des Selbstmordes in Kriegszeiten wurde daraus erklärt, daß das stolze Bewußtsein und die Hebung des Selbstgefühls, die der Kampf für höhere Güter gemeinhin weckt, dem Leben neuen Inhalt verleiht und daß der Schwung der nationalen Begeisterung auch lebensüberdrüssige Gemüter ergreift, mit fortreißt und mit neuem Lebensmut erfüllt. Schließlich ist es psychologisch begreiflich, daß das Leben für manchen erst dann an Reiz und Wert gewinnt, wenn wie im Krieg die Gefahr, es zu verlieren, in greifbare Nähe rückt.

Dieser Krieg, der in seinen Dimensionen alles bisher Dagewesene in den Schatten stellt, hat in so vieler Hinsicht Volkskörper und Volksseele nachhaltig beeinflußt, daß man sich eigentlich nicht wundern darf, wenn manche anscheinend bisher gesicherte Erfahrungstatsache ins Wanken gerät oder einer neuen Auffassung Platz machen muß. Die lange Dauer des Kampfes und ihre Rückwirkung auf die breiten Massen, die Schwierigkeiten der Ernährung sowie die Gefährdung und Vernichtung so vieler Existenzen, die mannigfachen Verluste an Gut und Blut und nicht zuletzt die Umwälzungen in der sozialen Struk- 
tur unseres Volkes haben den Enthusiasmus der ersten Kriegsmonate verdrängt und an seine Stelle das eiserne Pflichtbewußtsein zum Gebot der Stunde erhoben. Die mit der langen Dauer des Krieges immer fühlbarer werdenden Nöte haben somit ein die Selbstmordneigung in solchen Zeiten unterdrückendes Moment zu einem guten Teil in Wegfall gebracht. An Frontabschnitten, wo der Großkampf tobte oder die Gefechtstätigkeit eine lebhafte war, mag die Selbstmordziffer nach wie vor eine geringe gewesen sein, schon aus dem naheliegenden Grund, weil dort nur psychisch vollwertige Mannschaften Verwendung fanden und die psychische Einstellung auf Angriff und Abwehr etwaige Selbstmordgedanken einzelner in der kraftstrotzenden, lebensbejahenden Ümgebung nicht leicht Wurzel schlagen ließ. Hingegen war an solchen Frontteilen, die vorzugsweise auf den zermürbenden Stellungskampf angewiesen waren, wo größere Kampfhandlungen zu den Ausnahmen gehörten, mit einem stärkeren Herrortreten der Suicidneigung zu rechnen.

Bei einer bayrischen Landwehrdivision, die seit Beginn des Stellungskrieges einen verhältnismäßig ruhigen Frontabschnitt innehatte, wurden in diesem ganzen Zeitraum 15 erfolgreiche Selbstmordversuche registriert. Berücksichtigt man die Stärke dieser Division, deren Kopfzahl zeitweilig der eines Armeekorps gleichkam, so entsprach hier die Selbstmordziffer ungefähr den zahlenmäßigen Ergebnis, das wir hinsichtlich der Suicidneigung aus den Erfahrungen der Friedenszeiten gewonnen haben. Das Vorwiegen der älteren Jahrgänge, die die lange Trennung von Haus und Hof, von Weib und Kind besonders schwer empfanden, hatte zur Folge, daß in einem derart zusammengesetzten Truppenkörper die den Lebensüberdruß aufhebende Wirkung des Krieges nicht in Erscheinung trat.

Schließlich wurden an ruhigen Frontabschnitten in größerer Anzahl Mannschaften eingesetzt, die aus irgendeinem Grund wegen körperlicher oder geistiger Gebrechen nicht voll kriegsverwendungsfähig waren. Die Erhebungen über die Ursachen des Selbstmords im Feld wurden von seiten des zuständigen Kriegsgerichts vornehmlich von dem Gesichtspunkt aus gepflogen, ob bei den einzelnen Suicidhandlungen das Verschulden eines Dritten in Frage kam. In dieser Hinsicht wurde einwandfrei festgestellt, daß ein solcher Zusammenhang in jedem Fall auszuschließen war. Auch ungünstige häusliche Verhältnisse, insbesondere schlechter Vermögensstand, spielten ätiologisch bei dem selbstgewählten Tod der Heeresangehörigen keine Rolle. Nach dem Ausscheiden dieser zwei bedeutsamen Faktoren, deren eventuelle Wirksamkeit in der vorliegenden Frage zunächst eingehende Berücksichtigung verdient, ist es um so interessanter, den Gründen nachzugehen, die für den ernsten Schritt unserer Selbstmörder bestimmend 
gewesen sind. Daher seien in gedrängter Kürze die Umstände, unter denen jeweils das Suicid erfolgte, und die Nachrichten, die über die Personlichkeiten der Täter eingeholt werden konnten, im folgenden angeführt:

Fall 1: Unteroffizier G. Z., 34 Jahre alt, Jedig, Wobereitechniker. Fuhrung vorzuglich. Hat sich in seinem Quartier mit seinem Dienstgewehr erschossen. Schon zu Hause hochgradig nervös, schwärmte viel von der Erfindung eines Patents. Beim Ers.-Truppenteil ruhig und zurückgezogen; mied Gesellschaft; beim geringsten Anlaß sehr erregt. Wenn er hart angefahren wurde, konnte er kein Wort herausbringen. War erst zwei Monate im Feld; schrieb von draußen keinem seiner nächsten Angehorigen; war im Umgang wortkarg und zurückhaltend; hatte Angst vor den Strapazen des Feldzugs, fürchtete, er halte die langen Märsche nicht aus: entweder erschieße er sich oder mache etwas anderes; im Fall der Gefangennahme nehme er sich das Leben. Brütete stundenlang vor sich hin, antwortete kaum auf die Anrede seiner Kameraden; kurz vor seinem Tod besonders verstört.

Psychischer Befund: Depression.

Unmittelbare Veranlassung: Wahrscheinlich Furcht vor den strapazen des Kriegs.

Fall 2: Ldw. G. D., 34 Jahre alt, Gutler, verheiratet, Vater von vier Kindern. Führung gut. Hat sich in der Ortsunterkunft erhängt. Seit August 1914 im Heeresdienst. Gelegentlich eines Patrouillengangs, bei dem ein Mann fiel, ein anderer gefangengenommen wurde, blieb er, statt zu Hilfe zu eilen, im Schützengraben. Deshalb waren ihm von allen Seiten Vorwurfe gemacht worden. Doch war weder ein Verfahren gegen ihn eingeleitet noch ihm eine Bestrafung in Aussicht gestellt worden. Bildete sich ein, er komme vors Kriegsgericht; gab dieser Befürchtung unzählige Male Ausdruck. Beruhigenden Versicherungen von seiten seiner Vorgesetzten und Kameraden schenkte er keinen Glauben. Wurde von den Mannschaften wegen seiner steten Befurchtungen gehänselt. Ihn quälte noch ein peinigender Gedanke: Er hatte ein Notizbuch verloren, in dem er sich in unzulässiger Weise über militärische Dinge und Vorgesetzte organgen hatte. Fürchtete, daß dieses Notizbuch in fremde Hände geraten sei und er deshalb Strafe zu gewärtigen habe. Ließ sich auch diese ganzlich unbegrundete Sorge von seinen Kameraden nicht ausreden, denen er ständig seine Nơte weinend vorbrachte. Nach Angabe: seiner Ehefrau hatte er früher iber Kopfschmerzen geklagt, derart, daß er ,närrisch" zu werden fürchtete.

Psychischer Befund: Phobie.

Unmittelbare Veranlassung: Unbegrundete Furcht vor Ntrafe.

Fall 3: Musk. S. T., 21 Jahre alt, Arbeiter in einer chemischen Fabrik (Chemiker?), ledig. Führung recht gut. Hat sich mit seinem Dienstgewehr erschossen. Litt an Großmannsucht, lebte weit über seine Verhältnisse, unterhielt regen Briefwechsel mit Damen, ruhmte sich seiner Beziehungen zu guten Gesellschaftskreisen, glaubte sich zu etwas Höherem geboren, brustete sich mit seinen Erfolgen bei den Weibern, gab sich Kellnerinnen gegenüber als Doktor aus, legte sich fremde Namen und das Adelspradikat bei; sprach oft und viel über Selbstmord, was für ein schönes Gefühl man dabei empfinden müsse; er habe vor seinem Dienstantritt bereits rinen Selbstmordversuch mit Arsenik gemacht, er sei damals im Wald aufgefunden und ins Krankenhaus nach W. gebracht worden, sei in seinem Wahn mit einem Messer auf den Wärter losgegangen. Machte sich in den letzten Tagen einer wiederholten [Traubsuberschreitung schuldig. Soll zuletzt die Äußerung getan haben: 
„Ich kann meinem lieben, guten Leutnant nicht mehr unter die Augen treten, weil ioh gestern abend über den Urlaub geblieben bin!" Machte sich am Abend vor seinem Tod auch darüber Gedanken, daß er im Wirtshaus an seiner Zeehe 10 Pf. schuldig geblieben sei; das gehe gegen seine Ehre.

Psychischer Befund: Psychopathie.

Unmittelbare Veranlassung: Scham uber sein militarisches Vergehen und vielleicht Furcht vor der zu erwartenden Strafe.

Fall 4: Ldw. I. Z., 39 Jahre alt, Taglöhner, kinderlos verheiratet. Führung sehr gut. Hat sich in seinem Quartier erschossen. Seit August 1914 im Feld, klagte uber Schmerzen im Kopf, jammerte, daß niemand seinen Beschwerden Glauben schenke; seine Frau werde zu Hause vom Bürgermeister schikaniert. Sein Vater sei ins Irrenhaus gekommen. Er fühle sich hinsichtlich des Urlaubs zurüickgesetzt; deshalb sehr unglücklich. Sollte bei nächster Gelegenheit nach Hause fahren. Nach Erhebungen bei der Heimatbehörde war Z. immer ein aufgeregter, rabiater Mensch, namentlich wenn er getrunken hatte. Seine Klagen über die Schikane des Bürgermeisters entsprachen nicht den Tatsachen.

Psychischer Befund: Neurasthenie.

Unmittelbare Veranlassung: Vermeintliche Zurucksetzung beim Urlaub.

Fall 5: Ldw. M. W., 38 Jahre alt, Taglohner, verheiratet, Vater von drei Kindern. Führung sehr gut. Hat sich während des Urlaubs zu Hause erhängt. Seit August 1914 im Feld. Saß auf der Heimreise nachdenklich und sinnend im Eisenbahnwagen; früher lustig und gesprächig, beantwortete er jetzt kaum die Fragen seines Reisegefährten. Zu Hause angekommen, begab er sich ohne weiteres zur Ruhe. Kümmerte sich entgegen seiner früheren Gewohnheit nicht um seine Kinder, besuchte auch nicht seine Nachbarn, suchte fur sich allein zu bleiben, saß zu Hause, ohne zu sprechen, aß wenig, auch nicht seine Lieblingsspeisen, litt an Schlaflosigkeit. Auf Fragen gab er nur kurze Antworten; ging von zu Hause mit einem Rechen versehen weg und hängte sich im Walde auf. Lebte in glücklicher Ehe; vor dem Krieg trieb er sich tagelang umher, ohne daB seine Frau wußte, wo. Zwei Söhne einer Schwester seiner Mutter begingen ebenfalls Selbstmord durch Erhängen.

Psychischer Befund: Periodische (?) Depression.

Unmittelbare Veranlassung: Geistesstorung.

Fall 6: Trainsold. MI. K., 22 Jahre, Stallschweizer, ledig, hat sich in seinem Quartier mit seinem Dienstgewehr erschossen. Schrieb einen Brief an seine Eltern: „Liebe Eltern!... Mir ist nicht mehr gut auf der Welt. Näheres werdet ihr erfahren." Seit 8-10 Wochen verstimmt. lebte zurückgezogen. Machte sich Gedanken, weil er wegen der Pferdepflege vom Veterinär gerügt worden war, sprach vom Erschießen, wenn er kein anderes Pferd bekäme. Er gehe in den Wald, damit ihn niemand finde. Fiel auch seinen Quarticrleuten durch seine Schwermut und Selbstmordäußerungen auf.

Psychischer Befund: Depression.

Unmittelbare Veranlassung: Gekränkter Ehrgeiz infolge eines Tadels.

Fall 7: Ldst. Inf. G. W., 42 Jahre alt, Ofenarbeiter, verheiratet, hat sich im Wald bei $\mathrm{D}$. erhängt. War ein williger, aber geistig beschränkter Mann, der immer für sich allein blieb. Litt seit 8 Jahren an jährlich 2-3 mal wiederkehrenden epileptischen Anfällen. War ungeschickt, lieB sich die Briefe nach Hause von einem Kameraden schreiben; klagte darüber, da $B$ er beim Instruktionsunterricht nicht begreifen könne und von Kameraden gehänselt werde. ÄuBerte häufig Lebensüberdruß.

Psychischer Befund: Imbezillität und Epilepsie.

Unmittelbare Veranlassung: Insuffizienzgefühl. 
Fall 8: Ldst. H. R., 38 Jahre, ledig, Baner, hat sich im Verbindungsgraben mit seinem Dienstgewehr erschossen. Meldete sich häufig zum Arzt wegen Schlaflosigkeit und sonstiger nervöser Beschwerden; war daruber verstimmt, daß man ihm nicht recht Glauben schenke, er sci cin unglucklicher Mensch, ihm könne niemand helfen; unter einer Million sei nicht einer, der so schlimm daran sei wie er. Unterhielt sich häufig über religiösæe Dinge; wenn er keinen Halt mehr an Gott finde, werde er sich das Leben nehmen: sprach sonst wenig mit seinen Kameraden; zeigte zuletzt große Unruhe; 2 Tage vor seinem Tode verließ or trotz Minenbeschießung den Unterstand, anscheinend, um den Tod zu suchen. Seine stark religiös gefärbten Briefe zeigen schwermutigen Inhalt.

Psychischer Befund: Depression.

Unmittelbare Veranlassung: Hyporhondrische Beschwerden.

Fall 9: Unteroffizier J. H., 4) Jahre alt, Vorarbeitex, verheiratet, Vater von 6 Kindern. Seit Kriegsbeginn im Felde, hat viele Schlachten an der Verdunfront und in der Champagne mitgemacht, wurde ausgezeichnet. Hat sich in der Nähe der Ortsunterkunft mit seinem Dienstgewehr erschossen. Lebte immer zurickgezogen, schien zuweilen gedrückter Stimmung. Aus seinen hinterlassenen Briefen ging hervor, daß er sich bei der Urlaubserteilung zurückgesetzt fühlte.

Psychischer Befund: ?

Unmittelbare Veranlassung: Vermeintliche Zurücksetzung beim Urlaub.

Fall 10: Vizewachtm. R. K., 22 Jahre, stud. tech., ledig. In zwei hinterlassenen Briefen an seine Mutter schilderte er in ergreifender Weise seinen Seelenzustand: ,... im Verkehr mit anderen habe ich mich meist urterlegen gefühlt und immer wieder mußte ich empfinden, daß ich nirgends etwas galt. Meine Militärzeit hat mich in gesteigertem Maß mein Unvermögen empfinden lassen, bis ich jetzt an einem Punkt angelangt bin, wo sich meine Schwäche nicht mehr mit meiner Stellung vereinbaren läßt. Als ich Rekrut, Unteroffizier und selbst als ich Vizewachtmeister war, wurde mir die Schwäche nicht so fühlbar als jetzt, wo ich verantwortlicher Führer sein soll. Früher sollte ich nur gehorchen, jetzt soll ich des Gehorsams meiner Leute sicher sein, und das gelingt mir nicht . . . Mir fehlt die Kraft, mich durchzusetzen, infolgedessen auch Selbstvertrauen, Selbstbewußtsein, Stolz und Selbstsicherheit. Wie wird nun meine Zukunft nach dem Krieg aussehen? Ich werde stets der Sklave anderer sein ... Ich glaubte für Dich (seine Mutter) sorgen zu können, statt dessen falle ich Dir zur Last... ich habe auch nicht auf Besserung zu hoffen, deshalb bin ich schon langst zu einem Entschluß gekommen, den ich nicht in Verzweiflung, sondern mit ruhiger Überlegung erwogen ...."

„... Ich befinde mich in verzweifelter Lage; alle Geistesgegenwart hat mich verlassen; wenn es gilt, schnell und entschlossen zu uberlegen und zu handeln, versage ich vollkommen. Wenn mir jemand widerspricht, weiß ich nichts zu antworten. In meiner Verlegenheit halte ich mich einsam in meinem Quartier und bin fähig, stundenlang stumpfśinnig zu bruten. - Immer wieder dieselben Gedanken; mein Zustand muß wohl krankhaft sein...!" Hat sich in seinem Quartier mit dem Revolver erschossen.

Psychischer Befund: Depression (auf der Basis psychopathischer Veranlagung?).

Unmittelbare Veranlassung: Insuffizienzgefiuhl.

Fall 11: Ldw. St. S., verheiratet; hat sich auf dem Weg von seinem Ortsquartier zur Stellung erschossen. In letzter Zeit war er verdrießlich, unwillig und aufgeregt, da er uber den Lebenswandel seiner Frau unerfreuliche Nachrichten erhielt. Gegen N. schwebte ein Verfahren wegen erschwerten Ungehorsams. Bei 
der Vernehmung durch den Gerichtsoffizier wurde er sich offenbar erst der Tragweite seiner Handlung bewußt; aus dem zuruckgelassenen Schreiben geht hervor, daß er aus Furcht vor Strafe den Tod suchte.

Psychischer Befund: ?

Unmittelbare Veranlassung: Furcht vor Strafe.

Fall 12: Gefr. J. K., 31 Jahre alt, ledig, Dienstkneeht, seit August 1914 auf verschiedenen Kriegsschauplätzen. Von jeher beschränkt, manchmal verstórt, anscheinend infolge früherer Verletzung durch Kopfschuß. Ein Kamerad bezeichnet ihn als zeitweise geistig nicht normal. Äußerte eines Nachts in der Stellung ganz unvermittelt zu seinen Kameraden: „Heute nacht werde ich auf St. Peter geführt: da werden mir dic Augen verbunden und ich werde erschossen." Auf die Frage seiner Kameraden, was er damit meine, erfolgte keine Antwort mehr; gleich darauf krachte vielmehr der todliche Sichuß.

Psychischer Befund: Dämmerzustand?

Unmittelbare Veranlassung: Geistesstorung.

Fall 13: Feldw. F. K., 40 Jahre. Betriebsleiter, verheiratet, Vater von zwei Kindern, hat sich in seinem Quartier erschossen. War in der letzten Zeit infolge Arbeitsüberlastung sehr aufgeregt; machte sich Gedanken darüber, daß Material aus dem seiner Aufsicht unterstehenden Lager herausgekommen sei, für das er keine Belege hatte; furchtete deshalb verdächtigt zu werden. War ratlos und erregt, fiel einem befreundeten Kameraden um den Hals, fragte, was er tun solle. War ein fleißiger, gewissenhafter Arbciter, der sich nie etwas hatte zuschulden kommen lassen. War zwei Jahre zuvor wegen nervöser Überreizung und melancholischer Verstimmung in einer Heilanstalt.

Psychischer Befund: Nervosität. Depression?

Unmittelbare Veranlassung: Nervöse Überreizung.

Fall 14: Kan. H. B., Magisstratsoffiziant, 4l Jahre, verheiratet, Vater eines Kindes. Hat sich im Signalstand der Meldesammelstelle erhängt, nachdem er sich einen Brustschuß beigebracht hatte. War einige Tage zuvor vom Gerichtsoffizier in einem gegen ihn schwebenden Verfahren wegen sittlicher Verfehlungen vernommen worden. Soll sich als Beamter einer Jugendfürsorgestello an weiblichen Bureauangestellten und anderen seiner Obhut anvertrauten Personen in unsittlicher Weise vergangen haben. Auch wurden ihm Unregelmäßigkeiten in der Kassenfuhrung zur Last gelegt, so daß ihm Dienstentlassung drohte. Seit weiner Vernehmung einsilbig, gedrückt, grübelte viel, schickte Uhr, Geld und Messer nach Haus. In einem Brief an seine Frau stellt er entrustet die Beschuldigungen in Abrede, nimmt Abschied von Frau und Kind, segnet und tröstet sie.

Psychischer Befund: ?

Unmittelbare Veranlassung: Furcht vor Strafe.

Fall 15. Sold. F. S., 3I Jahre alt, Gastwirt, verheiratet, Vater von zwei Kindern, hat sich mit seinem Revolver erschossen. Ein in sich gekehrter, sehwermutiger Mensch; klagte über Schwindelgefuhl, jammerte, daß ihm im Gegensatz zu früher alles so schwer falle.

Psychischer Befund: Depression.

Unmittelbare Veranlassung: Nervose Beschwerden.

Ein flüchtiger Blick auf unsere Kasuistik genügt zur Feststellung der Tatsache, daß unsere Selbstmörder sich mit wenigen Ausnahmen aus Individuen rekrutieren, die nach ihrem Vorleben psychiatrisches Interesse verdienen. Es entspricht dieses Ergebnis durchats den Erfahrungen 
früherer Cntersucher (( ' a upp. Stelzuer), die den Nachweis führten, daß Selbstmordneigung fast stets auf der Basis einer psychisch krankhaften Veranlagung in Erscheinung tritt. Nur in drei Fällen unserer Ubersicht sind die anamnestischen Erhebungen zu dürftig, um einen Rückschluß auf diẹ geistige Eigenart der Täter zu gestatten. Bei allen übrigen sind die psychopathologischen Züge so hervorstechend, da $ß$ sie ganz von selhst auf die wechselseitigen Beziehungen zwischen Selbstmord und abnormem Seelenleben hinweisen. Soweit die mehr oder weniger skizzenhaften Fingerzeige aus dem Vorleben einen Einblick in die krankhaft veranderte Psyche gestatten. handelte es sich vorzugsweise um Depressionszustände verschiedener Art, sowie um psychopathische Konstitutionen und nervöse Erschöpfungszustände; in einem Fall (12), wo die suicidale Handlung direkt als Ausfluß einer akut in Firscheinung tretenden Geistesstörung zu betrachten ist, dürfte wohl ein Dämmerzustand die Tat ausgelöst haben. Die psychopathologischen Veranderungen sind nïcht ohne weiteres als Folgeerscheinung des Kriegs aufzufassen. sondern bei der Mehrzahl der Selbstmörder, soweit einigermaßen sichere Angaben zur Verfügung stehen, bereits in der früheren Vergangenheit deutlich erkennbar. Jedoch kann nicht in Abrede gestellt werden, daß die durch den Krieg geschaffenen Verhältnisse und Nöte die psychopathologische Eigenart der Selbstmörder in vielen Fällen erst an das Tageslicht gezerrt haben. Dies zeigt sich besonders deutlich bei der Betrachtung der besonderen Umstände, unter denen das Suicid begangen wurde, bzw. der unmittelbaren Veranlassung, die jeweils zur Ausführung der Tat geführt hat.

Der Selbstmörder, der in einer momentanen Zwangslage Hand an sich legt, weil er keinen anderen Ausweg sieht. verrat durch seine Handlung nicht immer eine psychische Minderwertigkeit. Erst die falsche Einschätzung der Situation, die Unfähigkeit. sich aus einseitigen Gedankengängen in die Wirklichkeit zurückzufinden, lenkt den Verdacht auf eine psychische Unzulänglichkeit. Nun schafft der Krieg, der die Persönlichkeit unterdrückt und von ihr ein Aufgehen in der Gesamtheit verlangt, begreiflicherweise fortgesetzt solche $Z$ wangslagen, unter denen der psychisch Minderwertige ungleich mehr leidet als der Vollsinnige. In solchen Situationen taucht der Gedanke an die Lebensflucht auf, schlägt Wurzel und wird zur Tat. um so sicherer, je länger der krankhaft Veranlagte der ihn beengenden, qualvollen Situation ausgesetzt ist. Die Motive, die unmittelbar zur Lebensflucht trieben, sind die gleichen, wie sie uns zum großen Teil aus den Friedenszeiten geläufig sind. Sie sind auch in unseren Fällen zumeist in der begründeten oder unbegrundeten Furcht vor Strafe und in dem Gefiuhl der Unfähigkeit zur Frfüllung der Forderungen des Tages zu erblicken. die der Dienst von der Leistungsfähigkeit des einzelnen verlangt. Daneben 
spielen gekränter Ehrgeiz und vermeintliche Zurücksetzmng eine nicht unwesentliche Rolle. Dem geistig Vollwertigen wird es psychologisch immer unerklärlich bleiben, daß der Heroismus. der sich zweifelsohne in der doch einen nicht geringen Grad von Selhstüberwindung verlangenden Vernichtung des eigenen Ichs offenbart, nicht genügt, $\mathrm{um}$ die Widerlichkeiten und Fährnisse des Daseins zu ertragen. Es läßt sich eben immer wieder nur - von wenigen Ausnahmen naturlich abgesehen - aus einer krankhaften Veranlagung oder Geistesrichtung erklären, wenn die Todessehnsucht stärker ist als die Lebensenergie. Menschlich begreiflich sind die Wälle von Selhsttötung. für die die treibende Kraft in langdanerndem Siechtum und qualvollem Leid zu suchen ist. Merkwürdigerweise ist die Zahl derjenigen, die aus solcher Ursache Selbstmord begehen, verschwindend klein. Die Motive für das Suicid im Felde sind fast ausnahmslos aus der Situation zu erklären, in und aus der sich psychisch minderwertige Individuen nicht zurechtfinden. 